\title{
The first initial-boundary value problem of parabolic Monge-Ampère equations outside a bowl-shaped domain
}

\author{
Limei Dai $^{1 *}$ and Huihui Cheng ${ }^{2}$
}

\section{"Correspondence:}

Imdai@wfu.edu.cn

${ }^{1}$ School of Mathematics and Information Science, Weifang University, Weifang, China Full list of author information is available at the end of the article

\section{Springer}

\begin{abstract}
In this paper, we study the parabolic Monge-Ampère equations $-u_{t} \operatorname{det}\left(D^{2} u\right)=g$ outside a bowl-shaped domain with $g$ being the perturbation of $g_{0}(|x|)$ at infinity. Under the weaker conditions compared with the problem outside a cylinder, we obtain the existence and uniqueness of viscosity solutions with asymptotic behavior for the first initial-boundary value problem by using the Perron method.
\end{abstract}

Keywords: Parabolic Monge-Ampère equations; Initial-boundary value problem; Bowl-shaped domain; Perron method; Asymptotic behavior

\section{Introduction}

Monge-Ampère equation is a class of fully nonlinear partial differential equations. The Dirichlet problem of elliptic Monge-Ampère equations on exterior domains is closely related to a celebrated result of Jörgens ( $n=2$ [1]), Calabi $(n \leq 5$ [2]), and Pogorelov ( $n \geq 2$ [3]). It asserts that any classical convex solution of elliptic Monge-Ampère equation

$$
\operatorname{det} D^{2} u=1 \quad \text { in } \mathbb{R}^{n}
$$

must be a quadratic polynomial. A simpler and more analytical proof was given by Cheng and Yau [4]. Caffarelli [5] proved that this result holds true for viscosity solutions. Then the result was extended to the Dirichlet problem of elliptic Monge-Ampère equation on exterior domains by Caffarelli and $\mathrm{Li}$ in [6] where the existence and uniqueness of the viscosity solutions were proved by the Perron method. Other results for elliptic Monge-Ampère equations on exterior domains can be referred to [7-11] and the references therein. The blow-up solutions to the Monge-Ampère equation and convex solutions of the MongeAmpère systems can be referred to $[12,13]$.

The Jörgens-Calabi-Pogorelov theorem for parabolic Monge-Ampère equation

$$
-u_{t} \operatorname{det} D^{2} u=1 \quad \text { in } \mathbb{R}^{n} \times(-\infty, 0]
$$

was established by Gutiérrez and Huang [14]. It is stated that if $u \in C^{4,2}\left(\mathbb{R}^{n} \times(-\infty, 0]\right)$ is a parabolically convex solution of (1.1) such that, for some positive constants $d_{1}, d_{2}$,

C) The Author(s) 2021. This article is licensed under a Creative Commons Attribution 4.0 International License, which permits use, sharing, adaptation, distribution and reproduction in any medium or format, as long as you give appropriate credit to the original author(s) and the source, provide a link to the Creative Commons licence, and indicate if changes were made. The images or other third party material in this article are included in the article's Creative Commons licence, unless indicated otherwise in a credit line to the material. If material is not included in the article's Creative Commons licence and your intended use is not permitted by statutory regulation or exceeds the permitted use, you will need to obtain permission directly from the copyright holder. To view a copy of this licence, visit http://creativecommons.org/licenses/by/4.0/. 
$-d_{1} \leq u_{t}(x, t) \leq-d_{2},(x, t) \in \mathbb{R}^{n} \times(-\infty, 0]$, then $u$ must be the form $u(x, t)=C t+P(x)$ with $C<0$ and $P$ being a convex quadratic polynomial. Then the Jörgens-Calabi-Pogorelov parabolic theorem was generalized to the equation $u_{t}=\rho\left(\log \operatorname{det} D^{2} u\right)$ with $\rho=\rho(z) \in$ $C^{2}(\mathbb{R})$ by Xiong and Bao [15], the equation $u_{t}-\operatorname{logdet} D^{2} u=f$ by Wang and Bao [16], and the equation $-u_{t} \operatorname{det} D^{2} u=f$ by Zhang, Bao, and Wang [17]. In [18], the author, using the Perron method, studied the first initial-boundary value problem for parabolic MongeAmpère equation outside a cylinder

$$
\begin{aligned}
& -u_{t} \operatorname{det} D^{2} u=g \quad \text { in }\left(\mathbb{R}^{n} \backslash \bar{\Omega}\right) \times(0, \tilde{T}], \\
& u=\phi(x, t) \quad \text { on } \partial \Omega \times[0, \tilde{T}], \\
& u=\psi(x) \quad \text { in }\left(\mathbb{R}^{n} \backslash \Omega\right) \times\{t=0\},
\end{aligned}
$$

where $u=u(x, t), x \in \mathbb{R}^{n}, t \in \mathbb{R}, u_{t}=\partial u / \partial t, D^{2} u$ is the Hessian matrix of $u$ with respect to the spatial variables $x, \tilde{T}>0$ and $\Omega$ is a smooth, bounded, and strictly convex open subset in $\mathbb{R}^{n}, g=g(x, t)=1+O\left(|x|^{-\alpha}\right),|x| \rightarrow \infty$ with $\alpha>2, \phi(x, t)$ and $\psi(x)$ are given continuous functions satisfying the compatibility condition. The existence and uniqueness of viscosity solutions with asymptotic behavior at infinity to (1.2)-(1.4) were obtained. The first initialboundary value problems of parabolic Monge-Ampère equations $u_{t}=\rho\left(\log \operatorname{det} D^{2} u\right)$ and $u_{t}-\log \operatorname{det} D^{2} u=f$ on exterior domains were also studied in [19-21]. Recently, the author and Bao [22] obtained the existence of entire solutions of the Cauchy problem for parabolic Monge-Ampère equations $-u_{t} \operatorname{det} D^{2} u=g$ with $g=g_{0}(|x|)+O\left(|x|^{-\alpha}\right)$ at infinity.

This kind of first initial-boundary value problem (1.2)-(1.4) on exterior domains is motivated by the interior problem of parabolic Monge-Ampère equations [23, 24]

$$
\left\{\begin{array}{l}
-u_{t} \operatorname{det} D^{2} u=g(x, t) \quad \text { in } \Omega \times(0, \tilde{T}], \\
u=\phi(x, t) \quad \text { on }(\partial \Omega \times[0, \tilde{T}]) \cup(\Omega \times\{t=0\}) .
\end{array}\right.
$$

In this paper, we study the parabolic Monge-Ampère equations $-u_{t} \operatorname{det} D^{2} u=g(x, t)$ with $g=g_{0}(|x|)+O\left(|x|^{-\alpha}\right)$ (see the following details for $g_{0}$ and $\alpha$ ) outside a bowl-shaped domain.

Let $D \subset \mathbb{R}^{n+1}$ be a bounded domain and $t \in \mathbb{R}$, define

$$
D(t)=\{x:(x, t) \in D\}
$$

Set $t_{0}=\inf \{t: D(t) \neq \emptyset\}$. The parabolic boundary of $D$ is defined by

$$
\partial_{p} D=\left(\overline{D\left(t_{0}\right)} \times\left\{t_{0}\right\}\right) \cup \bigcup_{t \in \mathbb{R}}(\partial D(t) \times\{t\})
$$

where $\bar{D}$ denotes the closure of $D$ and $\partial D(t)$ denotes the boundary of $D(t)$. The side boundary of $D$ is defined by $S D=\bigcup_{t \in \mathbb{R}}(\partial D(t) \times\{t\})$. The set $D \subset \mathbb{R}^{n+1}$ is called a bowl-shaped domain if for each $t, D(t)$ is convex and for $t_{1} \leq t_{2}, D\left(t_{1}\right) \subset D\left(t_{2}\right)$. One can also refer to [14].

Let $D$ be a bowl-shaped domain and $T=\sup \{t: D(t) \neq \emptyset\}, \mathbb{R}_{T}^{n+1}=\mathbb{R}^{n} \times\left(t_{0}, T\right]$. Then $S D=\partial D(t) \times\left[t_{0}, T\right]$. In the following, we shall abuse the notations $S D$ and $\partial D(t) \times\left[t_{0}, T\right]$. 
We shall consider the first initial-boundary value problem of parabolic Monge-Ampère equations

$$
\begin{aligned}
& -u_{t} \operatorname{det} D^{2} u=g(x, t) \quad \text { in } \mathbb{R}_{T}^{n+1} \backslash \bar{D}, \\
& u=\phi(x, t) \quad \text { on } \partial D(t) \times\left[t_{0}, T\right], \\
& u=\psi(x) \quad \text { in }\left(\mathbb{R}^{n} \backslash D\left(t_{0}\right)\right) \times\left\{t=t_{0}\right\} .
\end{aligned}
$$

Let $\tilde{D} \subset \mathbb{R}^{n+1}$, if for $(x, t) \in \tilde{D}$ a function $u$ is $2 k$ th continuous differentiable with spatial variables $x \in \mathbb{R}^{n}$ and $k$ th continuous differentiable with time variable $t$, we say that $u \in$ $C^{2 k, k}(\tilde{D})$. Let $U S C(\tilde{D})$ and $L S C(\tilde{D})$ be the sets of upper and lower semicontinuous realvalued functions on $\tilde{D}$, respectively. We say that a function $u \in U S C(\tilde{D})$ (or $L S C(\tilde{D}))$ is parabolically convex if $u$ is convex in $x$ and nonincreasing in $t$. The following definition of viscosity solutions is referred to [25].

Definition 1.1 Suppose that $u \in U S C\left(\mathbb{R}_{T}^{n+1} \backslash \bar{D}\right)\left(\operatorname{LSC}\left(\mathbb{R}_{T}^{n+1} \backslash \bar{D}\right)\right)$ is locally parabolically convex. We say that $u$ is a viscosity subsolution (supersolution) of (1.5) if for any function $\varphi \in C^{2,1}\left(\mathcal{N}_{r}(\bar{x}, \bar{t})\right.$ ) (with some $\mathcal{N}_{r}(\bar{x}, \bar{t}):=\left\{(x, t):|x-\bar{x}|<r, \bar{t}-r^{2}<t \leq \bar{t}\right\} \subset \mathbb{R}_{T}^{n+1} \backslash \bar{D}$, whenever

$$
u(x, t)-\varphi(x, t) \leq(\geq) u(\bar{x}, \bar{t})-\varphi(\bar{x}, \bar{t}) \quad \text { for any }(x, t) \in \mathcal{N}_{r}(\bar{x}, \bar{t})
$$

we must have

$$
-\varphi_{t}(\bar{x}, \bar{t}) \operatorname{det} D^{2} \varphi(\bar{x}, \bar{t}) \geq(\leq) f(\bar{x}, \bar{t}) .
$$

For the supersolution, we also need that $D^{2} \varphi(\bar{x}, \bar{t})>0$ in the matrix sense.

$u \in C^{0}\left(\mathbb{R}_{T}^{n+1} \backslash \bar{D}\right)$ is a viscosity solution of (1.5) if it is both a viscosity subsolution and supersolution of (1.5).

Definition 1.2 We say that $u \in U S C\left(\overline{\mathbb{R}_{T}^{n+1} \backslash D}\right)\left(L S C\left(\overline{\mathbb{R}_{T}^{n+1} \backslash D}\right)\right)$ is a viscosity subsolution (supersolution) of problem (1.5)-(1.7) if $u$ is a viscosity subsolution (supersolution) of (1.5), $u \leq(\geq) \phi(x, t)$ on $\partial D(t) \times\left[t_{0}, T\right]$, and $u \leq(\geq) \psi(x)$ for $(x, t) \in\left(\mathbb{R}^{n} \backslash D\left(t_{0}\right)\right) \times\left\{t=t_{0}\right\}$.

Then $u \in C^{0}\left(\overline{\mathbb{R}_{T}^{n+1} \backslash D}\right)$ is a viscosity solution of (1.5)-(1.7) if it is a viscosity solution of (1.5), $u=\phi(x, t)$ on $\partial D(t) \times\left[t_{0}, T\right]$, and $u=\psi(x)$ for $(x, t) \in\left(\mathbb{R}^{n} \backslash D\left(t_{0}\right)\right) \times\left\{t=t_{0}\right\}$.

We assume that $g$ and $\psi$ satisfy the following assumptions:

(G) $g \in C^{0}\left(\mathbb{R}^{n} \times\left[t_{0}, T\right]\right)$ is a positive function satisfying

$$
0<\inf _{\mathbb{R}^{n} \times\left[t_{0}, T\right]} g \leq \sup _{\mathbb{R}^{n} \times\left[t_{0}, T\right]} g<\infty
$$

and for the constant $\alpha>0$,

$$
g(x, t)=g_{0}(|x|)+O\left(|x|^{-\alpha}\right), \quad \text { uniformly for } t,|x| \rightarrow \infty,
$$

where $g_{0} \in C^{0}([0,+\infty))$ is positive,

$$
g_{0}(r)=O\left(r^{\beta}\right), \quad r \rightarrow+\infty,
$$


and $\beta$ is a constant, $\beta \geq-\alpha$,

$$
\frac{-n(\min \{\alpha, n\}-2)}{n-1}<\beta<\infty .
$$

( $\Psi$ ) Assume that there exists a constant $\gamma>0$ such that $\psi \in C^{0}\left(\mathbb{R}^{n} \backslash D\left(t_{0}\right)\right)$ satisfies in the viscosity sense

$$
\left\{\begin{array}{l}
\operatorname{det} D^{2} \psi=\frac{g\left(x, t_{0}\right)}{\gamma}, D^{2} \psi>0 \quad \text { in } \mathbb{R}^{n} \backslash \overline{D\left(t_{0}\right)}, \\
\psi=\phi\left(x, t_{0}\right) \quad \text { on } \partial D\left(t_{0}\right),
\end{array}\right.
$$

and for some $b \in \mathbb{R}^{n}$ and some constant $c, \psi(x)$ satisfies

$$
\begin{aligned}
& \limsup _{|x| \rightarrow \infty}|x|^{\min \{\alpha, n\}-2+\beta-\frac{\beta}{n}}\left|\psi(x)-\left(u_{0}(|x|)+b \cdot x+c\right)\right|<\infty, \quad \text { if } \alpha \neq n, \\
& \limsup _{|x| \rightarrow \infty}|x|^{n-2+\beta-\frac{\beta}{n}}(\ln |x|)^{-1}\left|\psi(x)-\left(u_{0}(|x|)+b \cdot x+c\right)\right|<\infty, \quad \text { if } \alpha=n,
\end{aligned}
$$

where

$$
u_{0}(|x|)=\left(\frac{n}{\gamma}\right)^{\frac{1}{n}} \int_{0}^{|x|}\left(\int_{0}^{s} z^{n-1} g_{0}(z) d z\right)^{\frac{1}{n}} d s
$$

is the solution of elliptic Monge-Ampère equations

$$
\operatorname{det} D^{2} u_{0}=\frac{g_{0}(|x|)}{\gamma}
$$

with $u_{0}(0)=0, u_{0}^{\prime}(0)=0$.

Our main result is as follows.

Theorem 1.1 Let D be a bowl-shaped domain in $\mathbb{R}^{n+1}, n \geq 3$, and SD be smooth and strictly convex. Assume that $g$ and $\psi$ satisfy $(G)$ and $(\Psi)$ respectively and $\phi \in C^{2,1}(\bar{D}), \phi$ is decreasing in $t$. Then, for the $b \in \mathbb{R}^{n}$ and the constant $c$ in (1.9) and (1.10), there exists a unique viscosity solution $u \in C^{0}\left(\overline{\mathbb{R}_{T}^{n+1} \backslash D}\right)$ of (1.5), (1.6), and (1.7) satisfying, for $t \in\left[t_{0}, T\right]$,

$$
\begin{aligned}
& \limsup _{|x| \rightarrow \infty}|x|^{\min \{\alpha, n\}-2+\beta-\frac{\beta}{n}}\left|u(x, t)-\left(-\gamma\left(t-t_{0}\right)+u_{0}(|x|)+b \cdot x+c\right)\right| \\
& \quad<\infty, \quad \text { if } \alpha \neq n,
\end{aligned}
$$

and

$$
\begin{aligned}
& \limsup _{|x| \rightarrow \infty}|x|^{n+\beta-2-\frac{\beta}{n}}(\ln |x|)^{-1}\left|u(x, t)-\left(-\gamma\left(t-t_{0}\right)+u_{0}(|x|)+b \cdot x+c\right)\right| \\
& <\infty, \quad \text { if } \alpha=n .
\end{aligned}
$$

So we extend the previous results [18-20] from $g \equiv 1$ or $g=1+O\left(|x|^{-\alpha}\right)$ to $g=g_{0}(|x|)+$ $O\left(|x|^{-\alpha}\right)$. Moreover, in the Dirichlet problem of elliptic Monge-Ampère equations on exterior domains, an important lemma (Lemma 5.1 [6]) is used to construct the viscosity 
subsolutions with asymptotic behavior. Similarly, for the parabolic Monge-Ampère equations, a viscosity subsolution with asymptotic behavior is needed to be constructed by an important lemma (Lemma $2.1[19]$ ) on a cylinder $Q=\Omega \times(0, \tilde{T}] \subset \mathbb{R}^{n+1}$. To construct the viscosity subsolutions of parabolic Monge-Ampère equations applying the lemma, we added the strong condition $\phi_{x_{i}, t}(x, t)=0$ for any $x \in \partial \Omega, 0 \leq t \leq \tilde{T}$ [18-20], which is not natural. In this paper, we establish a lemma on a bowl-shaped domain and then we use this lemma to construct the viscosity subsolutions without the strong condition $\phi_{x_{i}, t}(x, t)=0$.

This paper is arranged as follows. In Sect. 2, we give the important lemma on a bowlshaped domain with which the viscosity subsolution is constructed. Theorem 1.1 is proved in Sect. 3.

\section{An important lemma}

Lemma 2.1 Let D be a bowl-shaped domain in $\mathbb{R}^{n+1}$. Suppose that $S D$ is smooth and strictly convex and $\Phi(x, t) \in C^{2,1}(\bar{D})$. Then there exists some constant $C_{0}$, depending only on $n, \Phi$, $D$, such that, for any $(\xi, \lambda) \in S D, \xi \in \mathbb{R}^{n}, \lambda \in \mathbb{R}$, there exists $\bar{x}(\xi, \lambda, t) \in \mathbb{R}^{n+1}$ satisfying

$$
|\bar{x}(\xi, \lambda, t)| \leq C_{0}
$$

and

$$
v_{\xi, \lambda}(x, t)<\Phi(x, t) \quad \text { on } S D \backslash\{(\xi, \lambda)\}
$$

where, for $(x, t) \in \mathbb{R}^{n} \times\left[t_{0}, T\right]$,

$$
\nu_{\xi, \lambda}(x, t)=\Phi(\xi, t)+\frac{c_{*}}{2}\left(|(x, t)-\bar{x}(\xi, \lambda, t)|^{2}-|(\xi, \lambda)-\bar{x}(\xi, \lambda, t)|^{2}\right),
$$

and $c_{*}$ is any bounded positive constant.

In addition, for some positive constant $c_{0}$ and some bounded domain $D_{1} \subset \mathbb{R}^{n} \times\left[t_{0}, T\right]$, we have

$$
\frac{\partial v_{\xi, \lambda}}{\partial t}<-c_{0} \quad \text { in } D_{1}
$$

Proof Let $(\xi, \lambda) \in S D$, and $\Phi$ locally has the expansion

$$
\begin{aligned}
\Phi(x, t) & =\Phi(\xi, t)+(x-\xi) \cdot D_{x} \Phi(\xi, t)+\frac{1}{2}(x-\xi)^{\prime} D^{2} \Phi(\xi, t)(x-\xi) \\
& \geq \Phi(\xi, t)+(x-\xi) \cdot D_{x} \Phi(\xi, t)-\bar{C}|x-\xi|^{2}
\end{aligned}
$$

where $D_{x} \Phi$ is the gradient of $\Phi$ in $x, D^{2} \Phi$ is the Hessian matrix of $\Phi$ in $x,(\xi, t) \in \bar{D}$, and $\bar{C}=\frac{1}{2} \max _{\bar{D}}\left|D^{2} \Phi\right|$.

Let

$$
\bar{x}(\xi, \lambda, t)=-\frac{1}{c_{*}}\left(\Phi_{x_{1}}(\xi, t), \ldots, \Phi_{x_{n}}(\xi, t), 0\right)+\hat{c} v(\xi, \lambda)+(\xi, \lambda),
$$


where $v(\xi, \lambda)$ is the unit internal normal vector of $S D$ at $(\xi, \lambda)$ and $\hat{c}$ is sufficiently large but bounded positive constant to be determined. Then

$$
\begin{aligned}
\nu_{\xi, \lambda}(x, t)= & \Phi(\xi, t)+\frac{c_{*}}{2}\left(|(x, t)|^{2}-|(\xi, \lambda)|^{2}\right)-c_{*}(x-\xi, t-\lambda) \cdot \bar{x}(\xi, \lambda, t) \\
= & \Phi(\xi, t)+\frac{c_{*}}{2}|x-\xi|^{2}+\frac{c_{*}}{2}(t-\lambda)^{2} \\
& +(x-\xi) \cdot D_{x} \Phi(\xi, t)-c_{*} \hat{c}(x-\xi, t-\lambda) \cdot v(\xi, \lambda) .
\end{aligned}
$$

So

$$
\begin{aligned}
& \left(v_{\xi, \lambda}-\Phi\right)(x, t) \\
& \quad \leq \bar{C}|x-\xi|^{2}+\frac{c_{*}}{2}|x-\xi|^{2}+\frac{c_{*}}{2}(t-\lambda)^{2}-c_{*} \hat{c}(x-\xi, t-\lambda) \cdot v(\xi, \lambda) .
\end{aligned}
$$

By a translation, without loss of generality, we can assume that $\xi=0, \lambda=0$. Then

$$
\begin{aligned}
\nu_{\xi, \lambda}(x, t) & :=\tilde{v}(x, t) \\
& =\Phi(0, t)+\frac{c_{*}}{2}|x|^{2}+\frac{c_{*}}{2} t^{2}+x \cdot D_{x} \Phi(0, t)-c_{*} \hat{c}(x, t) \cdot v(0,0)
\end{aligned}
$$

and

$$
(\tilde{v}-\Phi)(x, t) \leq \bar{C}|x|^{2}+\frac{c_{*}}{2}|x|^{2}+\frac{c_{*}}{2} t^{2}-c_{*} \hat{c}(x, t) \cdot v(0,0) .
$$

We again rotate the coordinates to have $v(0,0)$ as one of the axes. That is, let $M$ be an orthogonal matrix such that $M e_{n+1}=v(0,0)$. Set $M(y, \gamma)=(x, t)$, then

$$
(\tilde{v}-\Phi)(x, t):=(\bar{v}-\bar{\Phi})(y, \gamma) \leq C_{1}|y|^{2}+C_{2} \gamma^{2}-c_{*} \hat{c} \gamma
$$

and

$$
\tilde{v}(x, t):=\bar{v}(y, \gamma) \leq C_{3}-c_{*} \hat{c} \gamma
$$

where $C_{1}, C_{2}$ are bounded and depend on $\bar{C}, c_{*}$ and $M, C_{3}$ is bounded and depends on $c_{*},\|\Phi\|_{C^{2,1}(\bar{D})}, M$ and $\operatorname{diam} D$. Since $S D$ is strictly convex, then $S D$ can be locally represented by

$$
\gamma=\rho(y)=O\left(|y|^{2}\right) .
$$

Thus, by (2.4),

$$
(\tilde{v}-\Phi)(x, t)=(\bar{v}-\bar{\Phi})(y, \gamma) \leq C_{1}|y|^{2}+C_{2} \rho^{2}(y)-\hat{c} c_{*} \rho(y) .
$$

Again by the fact that $S D$ is strictly convex, there exists a constant $\delta>0$ depending only on $D$ such that

$$
\rho(y) \geq \delta|y|^{2}, \quad \forall|y|<\delta .
$$


So

$$
\begin{aligned}
(\tilde{v}-\Phi)(x, t) & =(\bar{v}-\bar{\Phi})(y, \gamma) \\
& \leq C_{1}|y|^{2}+C_{2} \rho^{2}(y)-\hat{c} c_{*} \delta|y|^{2}, \quad \forall|y|<\delta .
\end{aligned}
$$

Clearly, by (2.6), for sufficiently large but bounded constant $\hat{c}$,

$$
(\tilde{v}-\Phi)(x, t)<0, \quad \forall 0<|y|<\delta, \gamma=\rho(y)
$$

where $\hat{c}$ depends only on $\delta,\|\Phi\|_{C^{2,1}(\bar{D})}, c_{*}$, and $M$.

On the other hand, by (2.7), we have

$$
\gamma \geq \delta^{3}, \quad \forall(y, \gamma) \in S D \backslash\{(y, \rho(y)):|y|<\delta\} .
$$

Then, for any $(y, \gamma) \in S D \backslash\{(y, \rho(y)):|y|<\delta\}$, by (2.5),

$$
\tilde{v}(x, t)=\bar{v}(y, \gamma) \leq C_{3}-\hat{c} \delta^{3} c_{*} .
$$

Choosing $\hat{c}$ large enough (depending only on $c_{*}, \delta$, $\left.\operatorname{diam} D,\|\Phi\|_{C^{2,1}(\bar{D})}, M\right)$ but still bounded, we get

$$
\tilde{v}(x, t)-\Phi(x, t)<0, \quad \forall(y, \gamma) \in S D \backslash\{(y, \rho(y)):|y|<\delta\} .
$$

From (2.3), we know that

$$
\frac{\partial v_{\xi, \lambda}}{\partial t}=\Phi_{t}(\xi, t)+c_{*}(t-\lambda)+(x-\xi) \cdot D_{x, t} \Phi(\xi, t)-c_{*} \hat{c} v_{n+1}(\xi, \lambda),
$$

where

$$
D_{x, t} \Phi(\xi, t)=\left(\frac{\partial^{2} \Phi}{\partial x_{1} \partial t}, \ldots, \frac{\partial^{2} \Phi}{\partial x_{n} \partial t}\right)^{\prime} .
$$

Therefore, for some positive constant $c_{0}$ and some bounded domain $D_{1} \subset \mathbb{R}^{n} \times\left[t_{0}, T\right]$, similar to the above arguments, by translation and rotation of the coordinates, we can choose $\hat{c}$ sufficiently large but bounded such that (2.2) holds. The lemma is proved.

Remark 2.1 By (2.2), it is easy to see that even if $\Phi_{x_{i}, t}(x, t) \not \equiv 0,(x, t) \in S D$, we still have $-\left(v_{\xi, \lambda}\right)_{t} \operatorname{det} D^{2} v_{\xi, \lambda} \geq g(x, t)$ in some bounded domain $D_{1}$. Then we avoid the bad condition $\Phi_{x_{i}, t}(x, t)=0$ for any $(x, t) \in S D$ in $[18-20]$.

\section{Proof of Theorem 1.1}

For the reader's convenience, we first give the following lemmas whose proof can be found in $[18,26]$.

Lemma 3.1 ([18]) Let $\Omega \subset \Omega_{1}$ be two open strictly convex subsets with smooth boundaries in $\mathbb{R}^{n}$ and $Q=\Omega \times\left(t_{0}, T\right], Q_{1}=\Omega_{1} \times\left(t_{0}, T\right]$. Suppose that $v \in C^{0}(\bar{Q})$ and $u \in C^{0}\left(\overline{Q_{1}}\right)$ are 
parabolically convex and satisfy respectively

$$
-v_{t} \operatorname{det} D^{2} v \geq f \quad \text { in } Q
$$

and

$$
-u_{t} \operatorname{det} D^{2} u \geq f \quad \text { in } Q_{1} .
$$

Furthermore,

$$
u \leq v \quad \text { in } Q, \quad u=v \quad \text { on } \partial \Omega \times\left[t_{0}, T\right] .
$$

Let

$$
w(x, t)= \begin{cases}v(x, t), & (x, t) \in Q, \\ u(x, t), & (x, t) \in Q_{1} .\end{cases}
$$

Then $w \in C^{0}\left(Q_{1}\right)$ is parabolically convex and satisfies, in the viscosity sense,

$$
-w_{t} \operatorname{det} D^{2} w \geq f \quad \text { on } Q_{1} \text {. }
$$

Lemma 3.2 ([26]) Let $\Omega_{1}$ be an open strictly convex subsets with smooth boundary in $\mathbb{R}^{n}$, $Q_{1}=\Omega_{1} \times\left(t_{0}, T\right]$, and $f \in C^{0}\left(Q_{1}\right)$ be nonnegative. Suppose that $\mathbb{S}_{0}$ is a nonempty family of subsolutions to the equation

$$
-u_{t} \operatorname{det}\left(D^{2} u\right)=f \quad \text { in } Q_{1} \text {, }
$$

and

$$
u(x, t)=\sup \left\{\omega(x, t) \mid \omega \in \mathbb{S}_{0}\right\}, \quad(x, t) \in Q_{1},
$$

then $u$ is a viscosity subsolution of (3.1).

Proof of Theorem 1.1 Through an affine transformation in the $x$-space and by subtracting a linear function to $u$, we may assume that $b=0$. The proof is divided into six steps.

Step 1. Construct a viscosity subsolution of (1.5), (1.6), (1.7).

Let $R>0, B_{R}(0)=\left\{x \in \mathbb{R}^{n}:|x|<R\right\}$. Without loss of generality, we may assume that $B_{2}(0) \subset \subset D\left(t_{0}\right)$. Let $R_{1}=\operatorname{diam}(D(T))$, then $D(T) \subset \subset B_{R_{1}}(0)$, choose $R_{2}>2 R_{1}$. Then $B_{2}(0) \subset \subset D\left(t_{0}\right) \subset \subset D(T) \subset \subset B_{R_{1}}(0) \subset \subset B_{R_{2}}(0)$. By Lemma 2.1, for any $(\xi, \lambda) \in \partial D(t) \times$ $\left[t_{0}, T\right]$, there exists $\bar{x}(\xi, \lambda, t) \in \mathbb{R}^{n+1},|\bar{x}(\xi, \lambda, t)|<\infty$ such that

$$
v_{\xi, \lambda}(x, t)<\phi(x, t), \quad(x, t) \in\left(\partial D(t) \times\left[t_{0}, T\right]\right) \backslash\{(\xi, \lambda)\},
$$

where

$$
\begin{aligned}
& \nu_{\xi, \lambda}(x, t) \\
& \quad=\phi(\xi, t)+\frac{c_{*}}{2}\left[|(x, t)-\bar{x}(\xi, \lambda, t)|^{2}-|(\xi, \lambda)-\bar{x}(\xi, \lambda, t)|^{2}\right], \quad(x, t) \in \mathbb{R}^{n} \times\left[t_{0}, T\right] .
\end{aligned}
$$


Then, by (2.2), we can choose $c_{*}$ large enough but bounded such that

$$
\begin{aligned}
& -\left(v_{\xi, \lambda}\right)_{t} \operatorname{det} D^{2} v_{\xi, \lambda} \geq \max _{(x, t) \in \overline{B_{R_{2}}(0)} \times\left[t_{0}, T\right]} g \geq g(x, t), \quad(x, t) \in B_{R_{2}}(0) \times\left(t_{0}, T\right], \\
& \operatorname{det} D^{2} v_{\xi, \lambda}\left(x, t_{0}\right) \geq g\left(x, t_{0}\right) / \gamma, \quad x \in B_{R_{2}}(0) .
\end{aligned}
$$

Set

$$
v(x, t)=\sup _{(\xi, \lambda) \in \partial D(t) \times\left[t_{0}, T\right]} v_{\xi, \lambda}(x, t), \quad(x, t) \in \mathbb{R}^{n} \times\left[t_{0}, T\right] .
$$

Then, by (2.1),

$$
v(x, t)=\phi(x, t), \quad(x, t) \in \partial D(t) \times\left[t_{0}, T\right]
$$

and by [27] and Lemma 3.2,

$$
\begin{aligned}
& -v_{t} \operatorname{det} D^{2} v \geq g(x, t), \quad(x, t) \in B_{R_{2}}(0) \times\left(t_{0}, T\right], \\
& \operatorname{det} D^{2} v\left(x, t_{0}\right) \geq g\left(x, t_{0}\right) / \gamma, \quad x \in B_{R_{2}}(0) .
\end{aligned}
$$

So

$$
\operatorname{det} D^{2} v\left(x, t_{0}\right) \geq \operatorname{det} D^{2} \psi(x), \quad x \in B_{R_{2}}(0) \backslash \overline{D\left(t_{0}\right)} .
$$

Choose two positive continuous functions $\bar{g}(|x|), \underline{g}(|x|)$ such that

$$
\gamma \bar{g}(|x|) \geq g(x, t) \geq \gamma \underline{g}(|x|)
$$

and

$$
\begin{array}{ll}
\gamma \underline{g}(|x|)=g_{0}(|x|)-c_{1}|x|^{-\alpha}, & |x| \rightarrow \infty, \\
\gamma \bar{g}(|x|)=g_{0}(|x|)+c_{2}|x|^{-\alpha}, & |x| \rightarrow \infty,
\end{array}
$$

where $c_{1}$ and $c_{2}$ are positive constants. For $a>0$, we define functions

$$
\begin{aligned}
u_{1}(x, t)= & -\gamma\left(t-t_{0}\right)+\inf _{B_{R_{1}} \times\left[t_{0}, T\right]} v \\
& +\int_{2 R_{1}}^{|x|}\left(\int_{1}^{s} n z^{n-1} \bar{g}(z) d z+a\right)^{\frac{1}{n}} d s, \quad(x, t) \in \mathbb{R}^{n} \times\left[t_{0}, T\right], \\
u_{2}(x, t)= & -\gamma\left(t-t_{0}\right)+\sup _{B_{R_{1}} \times\left[t_{0}, T\right]} v \\
& +\int_{2}^{|x|}\left(\int_{1}^{s} n z^{n-1} \underline{g}(z) d z+a\right)^{\frac{1}{n}} d s, \quad(x, t) \in \mathbb{R}^{n} \times\left[t_{0}, T\right] .
\end{aligned}
$$

Then $u_{1}, u_{2}$ are parabolically convex. Moreover,

$$
-\left(u_{1}\right)_{t} \operatorname{det} D^{2} u_{1}=\gamma \bar{g}(|x|) \geq g(x, t), \quad(x, t) \in\left(\mathbb{R}^{n} \backslash\{0\}\right) \times\left(t_{0}, T\right],
$$




$$
\begin{array}{ll}
-\left(u_{2}\right)_{t} \operatorname{det} D^{2} u_{2}=\gamma \underline{g}(|x|) \leq g(x, t), & (x, t) \in\left(\mathbb{R}^{n} \backslash\{0\}\right) \times\left(t_{0}, T\right], \\
\operatorname{det} D^{2} u_{1}(x, t)=\bar{g}(|x|) \geq g(x, t) / \gamma, & x \in \mathbb{R}^{n} \backslash\{0\}, t_{0} \leq t \leq T, \\
\operatorname{det} D^{2} u_{2}(x, t)=g(|x|) \leq g(x, t) / \gamma, & x \in \mathbb{R}^{n} \backslash\{0\}, t_{0} \leq t \leq T .
\end{array}
$$

For $|x| \leq R_{1}, t_{0} \leq t \leq T$,

$$
\begin{aligned}
u_{1}(x, t) & =-\gamma\left(t-t_{0}\right)+\inf _{B_{R_{1}} \times\left[t_{0}, T\right]} v+\int_{2 R_{1}}^{R_{1}}\left(\int_{1}^{s} n z^{n-1} \bar{g}(z) d z+a\right)^{\frac{1}{n}} d s \\
& \leq \inf _{B_{R_{1}} \times\left[t_{0}, T\right]} v \leq v(x, t) .
\end{aligned}
$$

We can choose $a_{0}>0$ such that, for $a \geq a_{0}$, the following three inequalities hold simultaneously:

$$
\begin{aligned}
u_{1}(x, t) & =-\gamma\left(t-t_{0}\right)+\inf _{B_{R_{1}} \times\left[t_{0}, T\right]} v+\int_{2 R_{1}}^{R_{2}}\left(\int_{1}^{s} n z^{n-1} \bar{g}(z) d z+a\right)^{\frac{1}{n}} d s \\
& \geq v(x, t), \quad \text { for }|x|=R_{2}, t_{0} \leq t \leq T, \\
u_{2}(x, t) & =-\gamma\left(t-t_{0}\right)+\sup _{B_{R_{1}} \times\left[t_{0}, T\right]} v+\int_{2}^{R_{2}}\left(\int_{1}^{s} n z^{n-1} \underline{f}(z) d z+a\right)^{\frac{1}{n}} d s \\
& \geq v(x, t), \quad \text { for }|x|=R_{2}, t_{0} \leq t \leq T, \\
u_{2}(x, t) & \geq \phi(x, t), \quad(x, t) \in \partial D(t) \times\left[t_{0}, T\right] .
\end{aligned}
$$

In addition, for $(x, t) \in \mathbb{R}^{n} \times\left[t_{0}, T\right]$, we have

$$
\begin{aligned}
u_{1}(x, t)= & -\gamma\left(t-t_{0}\right)+u_{0}(|x|)+v_{1}(a) \\
& -\int_{|x|}^{\infty}\left[\left(\int_{1}^{s} n z^{n-1} \bar{g}(z) d z+a\right)^{\frac{1}{n}}-\left(\int_{0}^{s} \frac{n}{\gamma} z^{n-1} g_{0}(z) d z\right)^{\frac{1}{n}}\right] d s,
\end{aligned}
$$

where the function $u_{0}(|x|)$ is $(1.11)$, and

$$
\begin{aligned}
\nu_{1}(a)= & \int_{2 R_{1}}^{\infty}\left[\left(\int_{1}^{s} n z^{n-1} \bar{g}(z) d z+a\right)^{\frac{1}{n}}-\left(\int_{0}^{s} \frac{n}{\gamma} z^{n-1} g_{0}(z) d z\right)^{\frac{1}{n}}\right] d s \\
& -u_{0}\left(2 R_{1}\right)+\inf _{B_{R_{1}} \times\left[t_{0}, T\right]} \nu .
\end{aligned}
$$

Then $v_{1}(a)$ is strictly increasing in $(0,+\infty)$ and

$$
\lim _{a \rightarrow+\infty} v_{1}(a)=+\infty
$$


Furthermore, by (1.8), we have $\beta+n>0$. Since $\bar{g}(z)=\frac{g_{0}(z)}{\gamma}+\frac{c_{2}}{\gamma} z^{-\alpha}, g_{0}(z)=O\left(z^{\beta}\right), z \rightarrow \infty$, we know that, as $s \rightarrow+\infty$,

$$
\begin{aligned}
& \left(\int_{1}^{s} n z^{n-1} \bar{g}(z) d z+a\right)^{\frac{1}{n}}-\left(\int_{0}^{s} \frac{n}{\gamma} z^{n-1} g_{0}(z) d z\right)^{\frac{1}{n}} \\
& =O\left(s^{1-\beta+\frac{\beta}{n}-\min \{\alpha, n\}}\right), \quad \text { if } \alpha \neq n,
\end{aligned}
$$

and

$$
\begin{aligned}
& \left(\int_{1}^{s} n z^{n-1} \bar{g}(z) d z+a\right)^{\frac{1}{n}}-\left(\int_{0}^{s} \frac{n}{\gamma} z^{n-1} g_{0}(z) d z\right)^{\frac{1}{n}} \\
& =O\left(s^{1-\beta+\frac{\beta}{n}-n} \ln s\right), \quad \text { if } \alpha=n .
\end{aligned}
$$

As a result, as $|x| \rightarrow \infty$,

$$
\begin{aligned}
& \int_{|x|}^{\infty}\left[\left(\int_{1}^{s} n z^{n-1} \bar{g}(z) d z+a\right)^{\frac{1}{n}}-\left(\int_{0}^{s} \frac{n}{\gamma} z^{n-1} g_{0}(z) d z\right)^{\frac{1}{n}}\right] d s \\
& \quad=\int_{|x|}^{\infty} O\left(s^{1-\beta+\frac{\beta}{n}-\min \{\alpha, n\}}\right) d s \\
& =O\left(|x|^{2-\beta+\frac{\beta}{n}-\min \{\alpha, n\}}\right), \quad \text { if } \alpha \neq n,
\end{aligned}
$$

and

$$
\begin{aligned}
& \int_{|x|}^{\infty}\left[\left(\int_{1}^{s} n z^{n-1} \bar{g}(z) d z+a\right)^{\frac{1}{n}}-\left(\int_{0}^{s} \frac{n}{\gamma} z^{n-1} g_{0}(z) d z\right)^{\frac{1}{n}}\right] d s \\
& =O\left(|x|^{2-\beta+\frac{\beta}{n}-n} \ln |x|\right), \quad \text { if } \alpha=n,
\end{aligned}
$$

where $2-\beta+\beta / n-\min \{\alpha, n\}<0$ by (1.8). Thus, as $|x| \rightarrow \infty$,

$$
u_{1}(x, t)=-\gamma\left(t-t_{0}\right)+u_{0}(|x|)+v_{1}(a)+O\left(|x|^{2-\beta+\frac{\beta}{n}-\min \{\alpha, n\}}\right), \quad \text { if } \alpha \neq n,
$$

and

$$
u_{1}(x, t)=-\gamma\left(t-t_{0}\right)+u_{0}(|x|)+v_{1}(a)+O\left(|x|^{2-\beta+\frac{\beta}{n}-n} \ln |x|\right), \quad \text { if } \alpha=n .
$$

Similarly, we can obtain that

$$
\begin{aligned}
u_{2}(x, t)= & -\gamma\left(t-t_{0}\right)+u_{0}(|x|)+\nu_{2}(a) \\
& -\int_{|x|}^{\infty}\left[\left(\int_{1}^{s} n z^{n-1} \underline{g}(z) d z+a\right)^{\frac{1}{n}}-\left(\int_{0}^{s} \frac{n}{\gamma} z^{n-1} g_{0}(z) d z\right)^{\frac{1}{n}}\right] d s,
\end{aligned}
$$

where

$$
\begin{aligned}
\nu_{2}(a)= & \int_{2}^{\infty}\left[\left(\int_{1}^{s} n z^{n-1} \underline{g}(z) d z+a\right)^{\frac{1}{n}}-\left(\int_{0}^{s} \frac{n}{\gamma} z^{n-1} g_{0}(z) d z\right)^{\frac{1}{n}}\right] d s \\
& -u_{0}(2)+\sup _{B_{R_{1}} \times\left[t_{0}, T\right]} v .
\end{aligned}
$$


It is clear that $v_{2}(a)$ is also strictly increasing in $(0,+\infty)$ and

$$
\lim _{a \rightarrow+\infty} v_{2}(a)=+\infty
$$

Then, as $|x| \rightarrow \infty$, we have

$$
\begin{cases}u_{2}(x, t)=-\gamma\left(t-t_{0}\right)+u_{0}(|x|)+v_{2}(a)+O\left(|x|^{2-\beta+\frac{\beta}{n}-\min \{\alpha, n\}}\right), & \text { if } \alpha \neq n, \\ u_{2}(x, t)=-\gamma\left(t-t_{0}\right)+u_{0}(|x|)+v_{2}(a)+O\left(|x|^{2-\beta+\frac{\beta}{n}-n} \ln |x|\right), & \text { if } \alpha=n .\end{cases}
$$

For the sufficiently large constant $c$ in (1.9) and (1.10), there exist $a_{1}(c)$ and $a_{2}(c)$ such that $v_{1}\left(a_{1}(c)\right)=v_{2}\left(a_{2}(c)\right)=c$. Thus, as $|x| \rightarrow \infty$, we have

$$
\begin{cases}u_{1}(x, t)=-\gamma\left(t-t_{0}\right)+u_{0}(|x|)+c+O\left(|x|^{2-\beta+\frac{\beta}{n}-\min \{\alpha, n\}}\right), & \text { if } \alpha \neq n, \\ u_{1}(x, t)=-\gamma\left(t-t_{0}\right)+u_{0}(|x|)+c+O\left(|x|^{2-\beta+\frac{\beta}{n}-n} \ln |x|\right), & \text { if } \alpha=n,\end{cases}
$$

and

$$
\begin{cases}u_{2}(x, t)=-\gamma\left(t-t_{0}\right)+u_{0}(|x|)+c+O\left(|x|^{2-\beta+\frac{\beta}{n}-\min \{\alpha, n\}}\right), & \text { if } \alpha \neq n, \\ u_{2}(x, t)=-\gamma\left(t-t_{0}\right)+u_{0}(|x|)+c+O\left(|x|^{2-\beta+\frac{\beta}{n}-n} \ln |x|\right), & \text { if } \alpha=n .\end{cases}
$$

So

$$
\lim _{|x| \rightarrow \infty}\left(u_{1}(x, t)-u_{2}(x, t)\right)=0, \quad t_{0} \leq t \leq T
$$

By (3.7), (3.8), (3.14) and the comparison principle, we obtain

$$
u_{1}\left(x, t_{0}\right) \leq u_{2}\left(x, t_{0}\right), \quad x \in \mathbb{R}^{n} \backslash B_{2}(0) .
$$

By (3.5), (3.6), (3.14), (3.15) and the comparison principle, we obtain

$$
u_{1}(x, t) \leq u_{2}(x, t), \quad(x, t) \in\left(\mathbb{R}^{n} \backslash B_{2}(0)\right) \times\left[t_{0}, T\right]
$$

For $a \geq a_{0}$, define

$$
\underline{u}_{a}(x, t)= \begin{cases}\max \left\{v(x, t), u_{1}(x, t)\right\}, & |x| \leq R_{2}, t_{0} \leq t \leq T \\ u_{1}(x, t), & |x| \geq R_{2}, t_{0} \leq t \leq T\end{cases}
$$

By (3.10), we know that $\underline{u}_{a} \in C^{0}\left(\mathbb{R}^{n} \times\left[t_{0}, T\right]\right)$. By Lemma 3.1, $\underline{u}_{a}$ satisfies in the viscosity sense

$$
-\left(\underline{u}_{a}\right)_{t} \operatorname{det} D^{2} \underline{u}_{a} \geq g(x, t), \quad(x, t) \in\left(\mathbb{R}^{n} \backslash\{0\}\right) \times\left(t_{0}, T\right]
$$

and

$$
\operatorname{det} D^{2} \underline{u}_{a}\left(x, t_{0}\right) \geq g\left(x, t_{0}\right) / \gamma=\operatorname{det} D^{2} \psi(x), \quad x \in \mathbb{R}^{n} \backslash \overline{D\left(t_{0}\right)} .
$$


As $|x| \rightarrow \infty$,

$$
\begin{cases}\underline{u}_{a}(x, t)=-\gamma\left(t-t_{0}\right)+u_{0}(|x|)+c+O\left(|x|^{2-\beta+\frac{\beta}{n}-\min \{\alpha, n\}}\right), & \text { if } \alpha \neq n, \\ \underline{u}_{a}(x, t)=-\gamma\left(t-t_{0}\right)+u_{0}(|x|)+c+O\left(|x|^{2-\beta+\frac{\beta}{n}-n} \ln |x|\right), & \text { if } \alpha=n .\end{cases}
$$

So

$$
\lim _{|x| \rightarrow \infty}\left(\underline{u}_{a}\left(x, t_{0}\right)-\psi(x)\right)=0 .
$$

In addition, we have, by (3.9) and (3.2),

$$
\underline{u}_{a}\left(x, t_{0}\right)=v\left(x, t_{0}\right)=\phi\left(x, t_{0}\right)=\psi(x), \quad x \in \partial D\left(t_{0}\right) .
$$

Thus, from the comparison principle, we know that

$$
\underline{u}_{a}\left(x, t_{0}\right) \leq \psi(x), \quad x \in \mathbb{R}^{n} \backslash D\left(t_{0}\right) .
$$

Moreover, thanks to (3.9) and (3.2),

$$
\underline{u}_{a}(x, t)=v(x, t)=\phi(x, t), \quad(x, t) \in \partial D(t) \times\left[t_{0}, T\right] .
$$

Then $\underline{u}_{a}$ is a viscosity subsolution of (1.5), (1.6), and (1.7).

By (3.4), (3.8), (3.11), (3.12) and the comparison principle,

$$
v\left(x, t_{0}\right) \leq u_{2}\left(x, t_{0}\right), \quad x \in \overline{B_{R_{2}} \backslash D\left(t_{0}\right)} .
$$

Then, by (3.3), (3.6), (3.11), (3.12), (3.19) and the comparison principle,

$$
v(x, t) \leq u_{2}(x, t), \quad(x, t) \in \overline{\left(B_{R_{2}} \times\left[t_{0}, T\right]\right) \backslash D} .
$$

So, combining with (3.16), we have

$$
\underline{u}_{a}(x, t) \leq u_{2}(x, t), \quad(x, t) \in \overline{\mathbb{R}_{T}^{n+1} \backslash D} .
$$

Step 2. Define the Perron solution of (1.5), (1.6), and (1.7).

Let $\mathcal{S}$ denote the set of locally parabolically convex functions $\omega \in C^{0}\left(\overline{\mathbb{R}_{T}^{n+1} \backslash D}\right)$ which are viscosity subsolutions of (1.5), (1.6), and (1.7) satisfying

$$
\omega(x, t) \leq u_{2}(x, t) .
$$

Then $\underline{u}_{a} \in \mathcal{S}$. So $\mathcal{S} \neq \emptyset$. Define

$$
u(x, t)=\sup \{\omega(x, t): \omega \in \mathcal{S}\}, \quad(x, t) \in \overline{\mathbb{R}_{T}^{n+1} \backslash D} .
$$

Step 3 . We prove that $u$ has the asymptotic behavior at infinity. 
On the one hand, by the definition of $u$, we get

$$
u(x, t) \leq u_{2}(x, t)
$$

Secondly, since $\underline{u}_{a} \in \mathcal{S}$, we get

$$
u(x, t) \geq \underline{u}_{a}(x, t) .
$$

By (3.13) and (3.17), we have

$$
\limsup _{|x| \rightarrow \infty}|x|^{\min \{\alpha, n\}+\beta-\frac{\beta}{n}-2}\left|u(x, t)-\left(-\gamma\left(t-t_{0}\right)+u_{0}(|x|)+c\right)\right|<\infty, \quad \text { if } \alpha \neq n,
$$

and

$$
\limsup _{|x| \rightarrow \infty}|x|^{n+\beta-\frac{\beta}{n}-2}(\ln |x|)^{-1}\left|u(x, t)-\left(-\gamma\left(t-t_{0}\right)+u_{0}(|x|)+c\right)\right|<\infty, \quad \text { if } \alpha=n .
$$

Step 4. We prove that $u(x, t)=\phi(x, t),(x, t) \in \partial D(t) \times\left[t_{0}, T\right]$, and $u\left(x, t_{0}\right)=\psi(x), x \in$ $\mathbb{R}^{n} \backslash D\left(t_{0}\right)$.

We first prove that $u\left(x, t_{0}\right)=\psi(x), x \in \mathbb{R}^{n} \backslash D\left(t_{0}\right)$. Since $\phi \in C^{2,1}(\bar{D})$, there exist some positive constants $q_{2} \geq q_{1}$ such that $-q_{2} \leq \phi_{t}(x, t) \leq-q_{1}$ on $\bar{D}$. Choose positive constants $p_{1}, p_{2}$,

$$
p_{1} \leq \min \left\{1, \frac{\gamma}{q_{1}}\right\}, \quad p_{2} \geq \max \left\{1, \frac{\gamma}{q_{2}}\right\}
$$

such that

$$
p_{1} q_{1} g\left(x, t_{0}\right) / \gamma \leq g(x, t), \quad p_{2} q_{2} g\left(x, t_{0}\right) / \gamma \geq g(x, t), \quad(x, t) \in \mathbb{R}_{T}^{n+1} \backslash \bar{D} .
$$

Let

$$
\begin{array}{ll}
\underline{U}(x, t)=-p_{2} q_{2}\left(t-t_{0}\right)+\psi(x), & (x, t) \in \overline{\mathbb{R}_{T}^{n+1} \backslash D}, \\
\bar{U}(x, t)=-p_{1} q_{1}\left(t-t_{0}\right)+\psi(x), & (x, t) \in \overline{\mathbb{R}_{T}^{n+1} \backslash D} .
\end{array}
$$

Then, in the viscosity sense,

$$
\begin{array}{ll}
-\underline{U}_{t} \operatorname{det} D^{2} \underline{U}=p_{2} q_{2} \operatorname{det} D^{2} \psi=p_{2} q_{2} g\left(x, t_{0}\right) / \gamma \geq g(x, t), \quad(x, t) \in \mathbb{R}_{T}^{n+1} \backslash \bar{D}, \\
-\bar{U}_{t} \operatorname{det} D^{2} \bar{U}=p_{1} q_{1} \operatorname{det} D^{2} \psi=p_{1} q_{1} g\left(x, t_{0}\right) / \gamma \leq g(x, t), \quad(x, t) \in \mathbb{R}_{T}^{n+1} \backslash \bar{D} .
\end{array}
$$

In addition, on $\partial D(t) \times\left[t_{0}, T\right]$,

$$
\begin{aligned}
\underline{U}(x, t) & =-p_{2} q_{2}\left(t-t_{0}\right)+\psi(x) \\
& =-p_{2} q_{2}\left(t-t_{0}\right)+\phi\left(x, t_{0}\right) \\
& \leq-q_{2}\left(t-t_{0}\right)+\phi\left(x, t_{0}\right) \\
& \leq \phi(x, t),
\end{aligned}
$$




$$
\begin{aligned}
\bar{U}(x, t) & =-p_{1} q_{1}\left(t-t_{0}\right)+\psi(x) \\
& =-p_{1} q_{1}\left(t-t_{0}\right)+\phi\left(x, t_{0}\right) \\
& \geq-q_{1}\left(t-t_{0}\right)+\phi\left(x, t_{0}\right) \\
& \geq \phi(x, t) .
\end{aligned}
$$

As $|x| \rightarrow \infty$,

$$
\lim _{|x| \rightarrow \infty}(\underline{U}(x, t)-u(x, t)) \leq 0
$$

and

$$
\lim _{|x| \rightarrow \infty}(\bar{U}(x, t)-u(x, t)) \geq 0
$$

Obviously, for $x \in \mathbb{R}^{n} \backslash \overline{D\left(t_{0}\right)}$,

$$
\underline{U}\left(x, t_{0}\right)=\bar{U}\left(x, t_{0}\right)=\psi(x) .
$$

Then $\underline{U}(x, t)$ and $\bar{U}(x, t)$ are viscosity subsolution and supersolution of (1.5), (1.6), and (1.7) respectively. So, $\underline{U} \in \mathcal{S}$. Moreover, for any $\omega \in \mathcal{S}$, we obtain $\omega(x, t) \leq \bar{U}(x, t)$. Thus

$$
\underline{U}(x, t) \leq u(x, t) \leq \bar{U}(x, t), \quad(x, t) \in \overline{\mathbb{R}_{T}^{n+1} \backslash D} .
$$

Therefore, $u\left(x, t_{0}\right)=\psi(x), x \in \mathbb{R}^{n} \backslash D\left(t_{0}\right)$.

Now we prove that $u(x, t)=\phi(x, t),(x, t) \in \partial D(t) \times\left[t_{0}, T\right]$. For any $\bar{\xi} \in \partial D(t), t_{0} \leq \bar{\gamma} \leq T$, on the one hand, since $\underline{u}_{a} \in \mathcal{S}$, then by (3.18),

$$
\liminf _{(x, t) \rightarrow(\bar{\xi}, \bar{\gamma})} u(x, t) \geq \lim _{(x, t) \rightarrow(\bar{\xi}, \bar{\gamma})} \underline{u}_{a}(x, t)=\phi(\bar{\xi}, \bar{\gamma}) .
$$

On the other hand, we have

$$
\limsup _{(x, t) \rightarrow(\bar{\xi}, \bar{\gamma})} u(x, t) \leq \phi(\bar{\xi}, \bar{\gamma}) .
$$

Indeed, for every $\omega \in \mathcal{S}$, we have

$$
\left\{\begin{array}{l}
-\omega_{t}+\Delta \omega \geq 0, \quad(x, t) \in\left(B_{R_{1}} \times\left(t_{0}, T\right]\right) \backslash \bar{D}, \\
\omega \leq \phi, \quad(x, t) \in \partial D(t) \times\left[t_{0}, T\right], \\
\omega \leq \bar{U}, \quad(x, t) \in\left(\left(B_{R_{1}} \backslash D\left(t_{0}\right)\right) \times\left\{t=t_{0}\right\}\right) \cup\left(\partial B_{R_{1}} \times\left[t_{0}, T\right]\right) .
\end{array}\right.
$$

Let $v^{+}$satisfy

$$
\left\{\begin{array}{l}
-v_{t}^{+}+\Delta v^{+}=0, \quad(x, t) \in\left(B_{R_{1}} \times\left(t_{0}, T\right]\right) \backslash \bar{D}, \\
v^{+}=\phi, \quad(x, t) \in \partial D(t) \times\left[t_{0}, T\right], \\
v^{+}=\bar{U}, \quad(x, t) \in\left(\left(B_{R_{1}} \backslash D\left(t_{0}\right)\right) \times\left\{t=t_{0}\right\}\right) \cup\left(\partial B_{R_{1}} \times\left[t_{0}, T\right]\right) .
\end{array}\right.
$$


By the comparison principle, $\omega \leq v^{+},(x, t) \in\left(\overline{\left.B_{R_{1}} \times\left[t_{0}, T\right]\right) \backslash D}\right.$. So $u \leq v^{+},(x, t) \in$ $\left(\overline{\left.B_{R_{1}} \times\left[t_{0}, T\right]\right) \backslash D}\right.$ and

$$
\limsup _{(x, t) \rightarrow(\bar{\xi}, \bar{\gamma})} u(x, t) \leq \lim _{(x, t) \rightarrow(\bar{\xi}, \bar{\gamma})} v^{+}(x, t)=\phi(\bar{\xi}, \bar{\gamma})
$$

Step 5. We prove that $u$ is a viscosity solution of (1.5).

As the proof of Theorem 1.4 in [21], we can prove that $u$ is a viscosity solution of (1.5). Step 6. We prove the uniqueness.

Suppose that $u$ and $v$ all satisfy (1.5), (1.6), (1.7), and (1.12) or (1.13). Then

$$
\lim _{x \rightarrow \infty}(u(x, t)-v(x, t))=0 .
$$

By the comparison principle, $u \equiv v,(x, t) \in \overline{\mathbb{R}_{T}^{n+1} \backslash D}$.

Theorem 1.1 is proved.

\section{Acknowledgements}

The authors are very grateful to the referees for their very helpful comments and suggestions.

\section{Funding}

The research was supported by the National Natural Science Foundation of China (No. 11201343) and Shandong Provincial Natural Science Foundation (ZR2018LA006).

\section{Abbreviations}

Not applicable.

\section{Availability of data and materials}

Not applicable.

\section{Ethics approval and consent to participate}

Not applicable.

\section{Competing interests}

The authors declare that they have no competing interests.

\section{Consent for publication}

Not applicable.

Authors' contributions

The first author contributed completely to the writing of the first manuscript and the second author edited the revised manuscript. All authors read and approved the final manuscript.

\section{Authors' information}

Not applicable.

\section{Author details}

'School of Mathematics and Information Science, Weifang University, Weifang, China. ${ }^{2}$ School of Mathematics and Statistics, North China University of Water Resources and Electric Power, Zhengzhou, China.

\section{Publisher's Note}

Springer Nature remains neutral with regard to jurisdictional claims in published maps and institutional affiliations.

Received: 27 November 2020 Accepted: 1 March 2021 Published online: 10 March 2021

\section{References}

1. Jörgens, K.: Über die Lösungen der Differentialgleichung $r t-s^{2}=1$. Math. Ann. 127, 130-134 (1954) (German)

2. Calabi, E.: Improper affine hyperspheres of convex type and a generalization of a theorem by K. Jörgens. Mich. Math. J. 5, 105-126 (1958)

3. Pogorelov, A.: On the improper convex affine hyperspheres. Geom. Dedic. 1, 33-46 (1972)

4. Cheng, S.Y., Yau, S.T.: Complete affine hypersurfaces, I. The completeness of affine metrics. Commun. Pure Appl. Math. 39, 839-866 (1986) 
5. Caffarelli, L.: Topics in PDEs: the Monge-Ampère equation. Graduate course, Courant Institute, New York University (1995)

6. Caffarelli, L., Li, Y.Y.: An extension to a theorem of Jörgens, Calabi, and Pogorelov. Commun. Pure Appl. Math. 56 549-583 (2003)

7. Bao, J.G., Li, H.G.: On the exterior Dirichlet problem for the Monge-Ampère equation in dimension two. Nonlinear Anal. 75, 6448-6455 (2012)

8. Bao, J.G., Li, H.G., Zhang, L.: Monge-Ampère equation on exterior domains. Calc. Var. Partial Differ. Equ. 52, 39-63 (2015)

9. Bao, J.G., Li, H.G., Zhang, L.: Global solutions and exterior Dirichlet problem for Monge-Ampère equation in $\mathbb{R}^{2}$. Differ Integral Equ. 29, 563-582 (2016)

10. Ju, H.J., Bao, J.G.: On the exterior Dirichlet problem for Monge-Ampère equations. J. Math. Anal. Appl. 405, 475-483 (2013)

11. Li, Y.Y., Lu, S.Y.: Existence and nonexistence to exterior Dirichlet problem for Monge-Ampère equation. Calc. Var. Partial Differ. Equ. 57, Paper No. 161 (2018)

12. Zhang, X.M., Feng, M.Q.: Boundary blow-up solutions to the Monge-Ampère equation: sharp conditions and asymptotic behavior. Adv. Nonlinear Anal. 9(1), 729-744 (2020)

13. Feng, M.Q.: Convex solutions of Monge-Ampère equations and systems: existence, uniqueness and asymptotic behavior. Adv. Nonlinear Anal. 10(1), 371-399 (2021)

14. Gutiérrez, C., Huang, Q.B.: A generalization of a theorem by Calabi to the parabolic Monge-Ampère equation. Indiana Univ. Math. J. 47, 1459-1480 (1998)

15. Xiong, J.G., Bao, J.G.: On Jörgens, Calabi, and Pogorelov type theorem and isolated singularities of parabolic Monge-Ampère equations. J. Differ. Equ. 250, 367-385 (2011)

16. Wang, B., Bao, J.G.: Asymptotic behavior on a kind of parabolic Monge-Ampère equation. J. Differ. Equ. 259, 344-370 (2015)

17. Zhang, W., Bao, J.G., Wang, B.: An extension of Jörgens-Calabi-Pogorelov theorem to parabolic Monge-Ampère equation. Calc. Var. Partial Differ. Equ. 57, Paper No. 90 (2018)

18. Dai, L.M.: Parabolic Monge-Ampère equations on exterior domains. Acta Math. Sinica (Chin. Ser.) 58, 447-456 (2015) (Chinese)

19. Dai, L.M.: Exterior problems for a parabolic Monge-Ampère equation. Nonlinear Anal. 100, 99-110 (2014)

20. Dai, L.M.: Exterior problems for more general parabolic Monge-Ampère equation in more general domain. J. Math. Anal. Appl. 427, 1190-1204 (2015)

21. Gong, S.Y., Zhou, Z.W., Bao, J.G.: Existence and uniqueness of viscosity solutions to the exterior problem of a parabolic Monge-Ampère equation. Commun. Pure Appl. Anal. 19, 4921-4936 (2020)

22. Dai, L.M., Bao, J.G.: Entire solutions of Cauchy problem for parabolic Monge-Ampère equations. Adv. Nonlinear Stud. 20, 769-781 (2020)

23. Lieberman, G.M.: Second Order Parabolic Differential Equations. World Scientific, River Edge (1996)

24. Wang, R.H., Wang, G.L.: On existence, uniqueness and regularity of viscosity solutions for the first initial-boundary value problems to parabolic Monge-Ampère equation. Northeast. Math. J. 8, 417-446 (1992)

25. Wang, R.H., Wang, G.L.: The geometric measure theoretical characterization of viscosity solutions to parabolic Monge-Ampère type equation. J. Partial Differ. Equ. 6, 237-254 (1993)

26. Dai, L.M.: Multi-valued solutions to a class of parabolic Monge-Ampère equations. Commun. Pure Appl. Anal. 13, 1061-1074 (2014)

27. Crandall, M., Ishii, H., Lions, P.: User's guide to viscosity solutions of second order partial differential equations. Bull. Am. Math. Soc. (N.S.) 27, 1-67 (1992)

\section{Submit your manuscript to a SpringerOpen ${ }^{\circ}$ journal and benefit from:}

- Convenient online submission

- Rigorous peer review

- Open access: articles freely available online

- High visibility within the field

- Retaining the copyright to your article

Submit your next manuscript at $\gg$ springeropen.com 